\title{
Prévision du transport solide sur un bassin versant. Application à l'envasement d'une retenue
}

\author{
S. Rais et M. Abidi \\ Ecole nationale d'ingénieurs de Tunis
}

\section{Introduction}

Parmi les problèmes posés par le transport solide sur les bassins versants sont cités : l'envasement des retenues et des cours d'eau, la diminution de la capacité utile des canaux d'irrigation, les migrations latérales des cours d'eau et en général leur instabilité.

L'envasement des retenues de barrages, en Tunisie, atteint des volumes élevés et pose ainsi de graves problèmes. Il est principalement dû aux taux élevés de l'érosion sur les bassins versants de ces barrages et à la sous-estimation des quantités de sédiments susceptibles de se déposer lors de leur conception (ABID et coll. [1], ClAUDE et coll. [7]). Il est donc impératif d'évaluer avec plus de précision les quantités de sédiments transportées du bassin versant et qui vont se déposer dans la retenue.

Plusieurs formules empiriques évaluant le taux d'érosion superficielle ont été développées dont celle de Musgrave (1947), l'Equation universelle de pertes en sols (1961), l'Equation universelle des pertes en sols modifiée (1972) (CHOw [5], WisCHMEIER et coll. [13]). Cependant ces formules ont été développées pour des bassins versants bien déterminés (situés surtout aux Etats-Unis d'Amérique) et elles font intervenir plusieurs coefficients qui varient dans de grands intervalles et qui sont difficiles à évaluer si on ne dispose pas de données précises et abondantes sur les volumes de sédiments transportés au niveau du bassin.

Par ailleurs ces formules empiriques évaluent surtout l'érosion superficielle du bassin (non totale). De plus il est difficile de prédire les processus dépendant du temps et de l'espace par des équations empiriques de régression.

Parmi les modèles mathématiques, décrivant le processus d'érosion des sols par l'eau, le modèle de Meyer et Wischmeier (1969, MEYER [11]) est basé sur des formules empiriques faisant intervenir de nouveau des coefficients qui sont difficiles à caler, en plus ce modèle n'est pas dynamique.

Dans cet article on présente un modèle mathématique permettant d'estimer les volumes de sédiments qui arrivent à la retenue. Ce modèle est dynamique basé sur les équations fondamentales (simplifiées) de l'écoulement et incorpore la plupart des caractéristiques du bassin versant, du barrage et hydrologiques. Une subdivision du bassin en sous-bassins permet de considérer chacun à part avec ses caractéristiques et non de considérer des coefficients globaux pour tout le bassin. Ce modèle est appliqué au cas d'un barrage tunisien (Chiba), et les résultats du modèle ont été comparés avec les mesures d'envasement effectuées sur cette retenue.

\section{Sediment transport prediction from a watershed. Application to reservoir silting}

This paper presents a numerical model enabling the estimation of the quantities of sediments transported from a watershed. The latter is subdivided into sub-watersheds composed of two sides drained by one single channel. For each sub-watershed the liquid mass is routed first on the two sides then along the channel, afterwards suitable sediment transport formulas are introduced to rout the sediments. An assemblage program manages the flow through all the watershed. The model takes into account its topography, the sediment size of its soils, the ground cover density and the hydrological data. The model gives good results compared to silting surveys made on a reservoir of the north of Tunisia. 


\section{Elaboration du modèle mathématique}

\subsection{Equations gouvernant le problème}

Le modèle est basé sur les équations fondamentales suivantes, pour les phases liquide et solide, et ce d'une part pour l'écoulement le long du cours d'eau et d'autre part pour l'écoulement superficiel :

\section{L'équation de continuité de l'eau :}

Le long du cours d'eau :

$$
\frac{\partial Q}{\partial x}+\frac{\partial A}{\partial t}=q_{t}
$$

où : $Q$ est le débit liquide, $A$ la section transversale du cours d'eau, $q_{l}$ le débit latéral par unité de longueur, $x$ l'abscisse, et $t$ le temps.

Pour l'écoulement superficiel : l'équation de continuité a la même forme que la précédente mais toutes ses variables sont considérées par unité de largeur de l'écoulement ( $q_{l}$ sera remplacé par le taux de ruissellement).

\section{L'équation d'énergie}

Pour les écoulements à pente relativement élevée, l'équation d'énergie a été approchée par celle d'une onde cinétique d'équation :

$$
S_{F}=S
$$

- pour l'écoulement le long du cours d'eau, $S_{F}$ est donné par la formule de Manning; d'où l'équation d'énergie devient : $S=n^{2} V^{2} / R^{4 / 3}(R:$ le rayon hydraulique, $V:$ la vitesse moyenne, $n:$ le coefficient de Manning, $S$ : la pente géométrique, $S_{F}$ : la pente de frottement) ;

- pour l'écoulement par voie de terre, $S_{F}$ est donné par la formule de Darcy-Weisbach, d'où l'équation d'énergie devient : $S=f V^{2} / 8 g R(f$ : le coefficient de frottement de Darcy-Weisbach, $g$ : l'accélération de la pesanteur).

\section{L'équation de continuité des sédiments :}

Pour l'écoulement le long du cours d'eau, elle s'écrit (simplifiée) :

$$
\frac{\partial Q_{s}}{\partial x}+(1-p) \gamma_{s} \frac{\partial(B z)}{\partial t}=q_{s l}
$$

où $Q_{s}$ est le débit solide total, $p$ la porosité du lit, $\gamma_{s}$ le poids volumique des sédiments, $B$ la largeur du lit, $z$ la cote à l'abscisse $x$, et $q_{s l}$ le débit solide latéral par unité de longueur.

Pour l'écoulement par voie de terre, l'équation est similaire à l'équation (3), mais elle est considérée par unité de largeur du versant, et le débit latéral est nul.

Formules de transport solide utilisées:

Le transport solide se fait par charriage et en suspension et pour chaque mode de transport il existe dans la littérature (CHOw [5], LARRAS [9], SimONS et coll. [12] ...) une multitude de formules basées sur des approches déterministe ou stochastique du problème de transport.

Le choix d'une formule appropriée devient encore difficile quand on ne dispose pas, comme c'est le cas, de mesures des quantités transportées dans différents sites du bassin versant à étudier. Par ailleurs ces formules ont été vérifiées dans d'autres pays, mais on ne connaît pas assez bien leur performance sur des cas tunisiens. Cependant on a choisi pour cette étude :

- la formule de Meyer-Peter et Muller (1948) pour le charriage,

- la formule de Vélikanov (1953) pour la suspension.

Ceci puisque ces formules conviennent assez bien aux caractéristiques du bassin versant étudié, englobent la plupart des paramètres de l'écoulement, sont d'une application facile, et ont donné des résultats acceptables pour un cas tunisien (Oued Zéroud, BACcouche [2]).

La formule de Meyer-Peter et Muller se base sur une approche énergétique et donne le débit solide pondéral $q_{s}$ par unité de largeur du lit comme :

$$
q_{s}=K\left(\tau-\tau_{c}\right)^{3 / 2} ; \quad \text { où : } \quad K=\frac{8}{\rho^{1 / 2}} \frac{\gamma_{s}}{\gamma_{s}-\gamma}
$$

$\tau_{c}$ est l'effort tranchant critique sur le lit, approché par

$$
\tau_{c}=0,047\left(\gamma_{s}-\gamma\right) D_{50}
$$

$\tau=\gamma R S\left(k_{s} / k_{r}\right)^{3 / 2}$ est l'effort tranchant moyen effectif ; avec: $D_{50}$ : le diamètre médian des sédiments, $\gamma:$ le poids volumique de l'eau, $\left(k_{s} / k_{r}\right)^{3 / 2}:$ un terme correctif de la rugosité ( $k_{s}$ et $k_{r}$ sont les coefficients de Strickler dus respectivement à la rugosité globale du lit et à celle des particules seules) et $\rho$ : la masse volumique de l'eau.

La formule de Vélikanov du transport en suspension se base sur une approche énergétique, et s'applique mieux aux rivières larges à lit sableux. Simplifiée cette formule évalue le débit solide comme :

$$
Q_{s}=\eta\left(\frac{\rho \rho_{s}}{\rho_{s}-\rho} \cdot \frac{V}{W} \cdot S\right) \times Q
$$

où : $W$ est la vitesse de chute moyenne d'une particule solide considérée et $\eta$ est le nombre de Vélikanov traduisant la capacité de transport de l'écoulement et varie pour un écoulement naturel de $7 \times 10^{-4}$ à $7 \times$ $10^{-3}$.

\subsection{Principales hypothèses simplificatrices adoptées}

Le transport éolien est considéré négligeable devant le transport hydrique.

Subdivision et représentation du bassin versant : afin de permettre une simulation numérique de sa topographie, le bassin versant (pour le cas d'application, représenté par la figure 1) est subdivisé en sous-bassins, de façon à ce que chacun d'eux soit formé de deux versants, drainés par un seul cours d'eau ; chaque versant est représenté par son rectangle équivalent. La pente moyenne du versant est prise comme la moyenne arithmétique des pentes de quelques segments, normaux à son contour (LINSLEY et coll. [10]). Les sections droites des cours d'eau sont assimilées à des sections rectangulaires larges.

Assimilation des quantités de sols érodés à la capacité de transport: le modèle peut surestimer les volumes érodés des sols du bassin, puisqu'on a supposé que le 

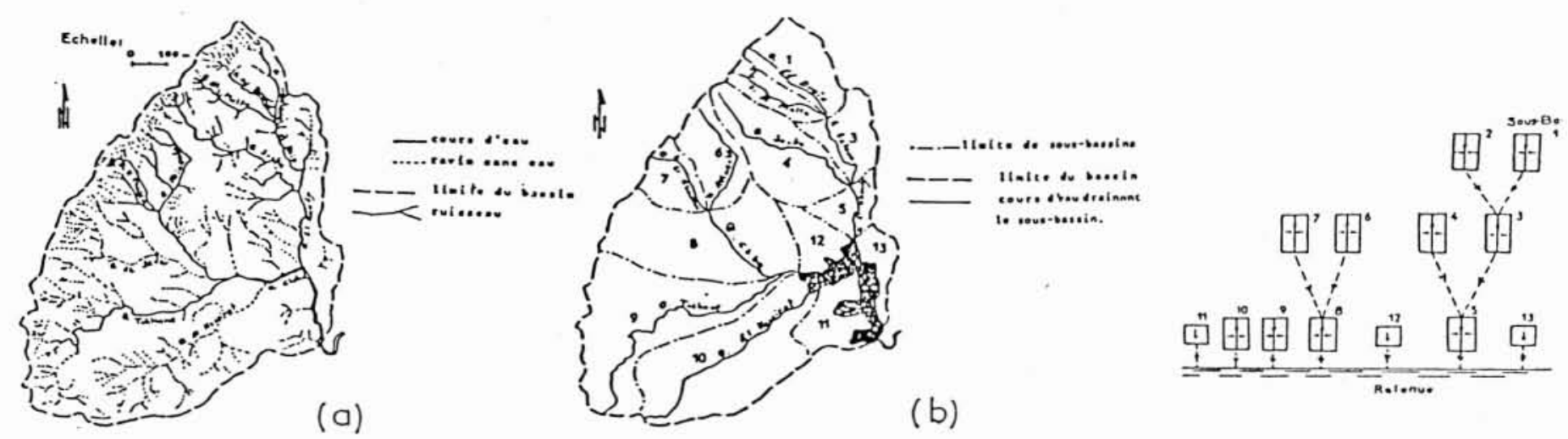

1. Réseau hydrographique (a). Subdivision du bassin versant de l'oued Chiba en sous-bassins (b).

2. Schéma d'assemblage de l'écoulem. dans le bassin versant.

cours d'eau et versants charrient leur capacité de transport. Ainsi on n'évalue pas les quantités de sédiments qui ont été arrachées aux sols du bassin, puisqu'on ne dispose pas de mesures sur terrain (pour le cas étudié) pour caler les formules empiriques évaluant ces quantités.

\subsection{Formulation du modèle}

Pour la procédure de résolution, en premier lieu les équations de continuité de l'eau et d'énergie sont résolues par la méthode des différences finies explicite, d'abord pour l'écoulement superficiel sur les versants puis pour celui le long du cours d'eau. Ensuite les débits solides sont calculés (pour chaque pas de temps et d'espace) à l'aide des formules de transport solide, des résultats de l'acheminement de l'eau précédemment achevé et des caractéristiques du bassin versant. En dernier lieu, l'équation de continuité des sédiments est résolue par la même méthode, afin de déterminer les changements de niveau que subit le lit.

Les équations précédentes étant résolues pour chaque sous-bassin, un programme d'assemblage permet de les regrouper (spécifiant leurs conditions aux limites), et d'ordonner l'écoulement dans le bassin entier. Le schéma d'assemblage est donné par la figure 2 pour le cas traité.

La condition de stabilité adoptée pour la méthode de résolution numérique est celle de Courant-FriedrichsLévy.

\section{Cas d'application}

Le modèle élaboré a été appliqué au bassin versant (couvrant $64 \mathrm{~km}^{2}$ ) du barrage Chiba, qui est un barrage moyen en terre.

\subsection{Pédologie et géologie du bassin versant}

Sa limite Sud-Est est formée de piedmonts de montagnes et de collines, constitués principalement de sols sableux à pentes raides (dépassant parfois $10 \%$ ), facilement attaqués par l'érosion. A l'Ouest le bassin est limité par une chaîne de montagnes constituées principalement de grès calcaire et non calcaire. A l'intérieur, il est formé de plaines à sols argileux profonds (CHAUVEL [4]) les pentes des versants variant en moyenne de 3 à $5 \%$.

\subsection{Caractéristiques hydrologiques du bassin versant}

Le réseau hydrographique : il est formé principalement dans sa partie centrale et Sud-Ouest par l'Oued Chiba et ses effluents et dans sa partie Nord par l'Oued El Khoudji et ses effluents (figure 1).

Le climat: il se distingue principalement par deux saisons, une humide (s'étalant de novembre jusqu'à la fin de mars) et une saison sèche.

Les pluies: la pluviométrie moyenne annuelle de la région de Chiba est de l'ordre de $400 \mathrm{~mm}$. Les pluies fines de moyenne journalière inférieure à $5 \mathrm{~mm}$ sont les plus fréquentes (tombant 50 sur 73 jours de pluie par an).

\subsection{Paramètres liés à l'envasement de la retenue}

Le temps de rétention: c'est le temps moyen qu'une particule d'eau reste dans le réservoir, évalué comme le rapport de capacité de la retenue au flux entrant. Pour le cas de Chiba, il est estimé en moyenne à 1,63 an.

Le coefficient d'interception, $C_{I}$, représentant la capacité d'un réservoir à piéger les sédiments qui y entrent, est exprimé comme le pourcentage de l'apport des sédiments qui est retenu dans le réservoir.

Plusieurs abaques et formules empiriques (BRUNE et ALLEN, 1941 ; CHURCHILl, 1948...) évaluant $C_{I}$ existent dans la littérature (BORLAND [3], CHOw [5], ...) ; pour Chiba il est estimé à $90 \%$.

\subsection{Résultats des campagnes de mesures de l'envasement}

Trois campagnes de mesures d'envasement (par échosondeur à ultrasons) ont été effectuées sur la retenue du barrage Chiba, l'une en 1975 (CLAUDE et coll. [7]), l'autre en août 1981 (ABID et coll. [1]), et la troisième, effectuée dans le cadre de cette étude, en 1987. Les résultats des deux dernières campagnes de mesures sont plus proches que ceux de la première et ont donné un apport solide annuel moyen de $128000 \mathrm{~m}^{3}$, ce qui correspond à un taux de comblement moyen de $1,27 \%$.

Les volumes de sols arrachés du bassin versant sont obtenus à partir des volumes déposés dans la retenue en tenant compte de son coefficient d'interception et des volumes de sédiments déversés et dévasés. 


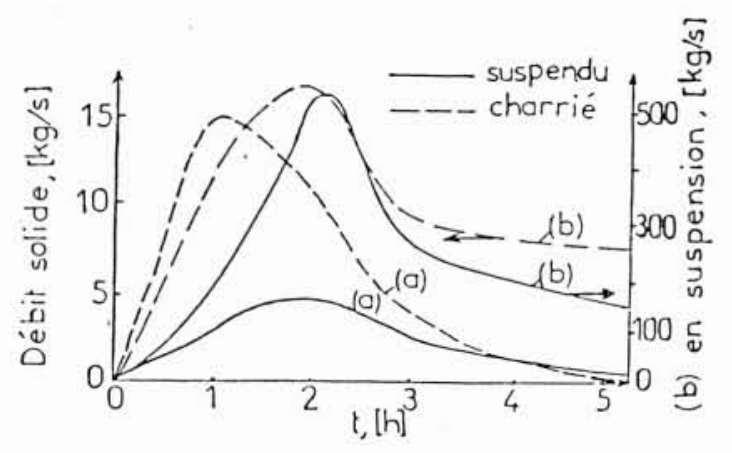

3. Variation, avec le temps, des débits solides charriés et en suspension simulés (pour un versant (a) et pour le cours d'eau $(b))$.

Afin de convertir les volumes de sédiments en poids on a tenu compte de la consolidation des matériaux déposés dans la retenue à l'aide de formules empiriques dont celle de Miller C. R. (BorLand [3]).

\subsection{Données du modèle}

Granulométrie des sols: des échantillons prélevés et analysés, au cours de cette étude dans les oueds ainsi qu'en quelques points du bassin, ont révélé que les sols sont essentiellement formés d'argile, de silt et de sable fin et moyen. Pour le modèle, la granulométrie des sols de chaque versant et cours d'eau est représentée par 3 ou 4 diamètres caractéristiques.

Caractéristiques géométriques des sous-bassins: chaque versant d'un sous-bassin est caractérisé par sa largeur, et par sa pente moyenne et chaque cours d'eau par sa longueur, sa pente moyenne et sa largeur.

Rugosité des cours d'eau et versants: pour le cours d'eau elle est représentée par le coefficient de Manning $n$, incorporant les effets de la nature du sol, de la végétation, des irrégularités de surface et du degré de courbature (formule de Cowan).

Pour les versants, la rugosité est représentée par le coefficient de Darcy-Weisbach $f$. Les valeurs de $f$ sont données par des abaques (CHOw [6]) pour différents types de canaux ayant des rugosités faibles à moyennes et en fonction du régime d'écoulement. Par conséquent, pour les versants partiellement couverts par la végétation ou d'autres rugosités de dimensions importantes, ces valeurs de $f$ ont été pondérées par le pourcentage du couvert végétal.

Données hydrologiques: ce sont principalement les distributions du taux de ruissellement dans le temps, pour les crues considérées.

\section{Résultats du modèle et discussion}

Deux années hydrologiques ont été simulées par le modèle (1981-82 et 1983-84). Des tests de sensibilité sur les coefficients de frottement ont montré l'importance de $f$ sur le transport solide et la nécessité de raffiner sa détermination, mais le rapport $k_{s} / k_{r}$ n'est pas très déterminant. D'autre part dans l'équation de Vélikanov du transport en suspension on a testé toutes les valeurs que peut prendre $\eta$. La valeur la plus appropriée a été trouvée : $\eta=0,0021$, d'ailleurs c'est cette même valeur qui a été adoptée dans l'étude effectuée sur l'Oued Zéroud (BACCOUCHE [2]).

Le volume de sédiments transportés du bassin, simulé par le modèle, a été trouvé assez proche, mais légèrement inférieur (de 10 à $20 \%$ ) au volume annuel moyen de sédiments atteignant la retenue. Ceci est dû en partie au fait qu'au cours des simulations on n'a pas considéré toutes les averses de l'année (ôtant celles à faible intensité), et d'autre part aux erreurs commises (estimées à $10 \%$ ) sur les mesures d'envasement.

En considérant les volumes transportés par unité de surface de chaque sous-bassin, on a pu détecter ceux qui lâchent plus de sédiments à la retenue. Quelques résultats simulés par le modèle sont présentés par les figures 3 et 4, elles correspondent à une averse de l'année 1983-84 dont l'intensité de pluie cause le taux de ruissellement présenté sur la figure 4.

La figure 3 donne les variations, en fonction du temps, des débits solides suspendu et charrié, pour le sousbassin $\mathrm{N}^{\circ} 5$.

La figure 4 présente aussi, pour la même averse et sous-bassin l'hydrogramme et le sédimentogramme (débit solide total) simulés pour le cours d'eau.

On constate que : pour les versants, les débits solides charriés sont supérieurs à ceux en suspension, ceci est dû à ce que la lame d'eau s'y écoulant est faible et que l'écoulement est considéré laminaire (pour la plupart des versants) ; ce qui fait que la suspension, qui est due en grande partie à la turbulence de l'écoulement, est faible.

Pour le cours d'eau, le débit solide en suspension est au contraire beaucoup plus grand que celui charrié, ceci est dû à ce que les tirants d'eau et par suite les débits liquides sont importants pour le cours d'eau et que l'écoulement y est turbulent (dans la plupart des cas).

Enfin on a pu trouver, à l'aide du modèle développé, la fréquence et l'intensité de la précipitation qui causerait à elle seule le volume de transport solide moyen pour toute l'année. C'est l'averse d'intensité décennale d'une heure de durée (d'après les courbes d'intensité-duréefréquence développées pour une région voisine, КСHOUK et coll. [8]). 


\section{Conclusion}

Le modèle numérique développé est dynamique permettant d'estimer l'évolution, au cours de chaque averse considérée, des volumes de sédiments transportés, d'un bassin-versant à une retenue aménagée sur celui-ci et de déceler les régions dans le bassin ayant les plus grandes capacités de transport, et qui devraient être stabilisées en priorité.

Malgré les simplifications adoptées, qui sont pour la plupart justifiées, le modèle a pu estimer les volumes de sédiments transportés à la retenue de Chiba, ceux-ci ont été vérifiés par des mesures d'envasement effectuées sur celle-ci. Ce modèle pourrait être amélioré si on disposait de mesures de transport solide effectuées sur le bassin versant.

Le modèle a été appliqué à un cas de bassin versant moyen mais il peut être appliqué à des bassins plus grands ou plus petits, moyennant une subdivision et un programme d'assemblage appropriés.

Enfin l'averse unique représentant une année hydrologique moyenne, du point de vue du transport solide, a été trouvée d'intensité décennale, d'une heure de durée. Ceci permet d'estimer le volume solide annuel moyen transporté à la retenue en l'absence d'autres données hydrologiques.

\section{Références}

[1] AвID A., ZaHAF H. - « Envasement et Dévasement des Retenues de Barrages en Tunisie ", Revue Tunisienne de l'Equipement, octobre-novembre-décembre 1981, $\mathrm{N}^{*} 38$.

[2] BACCOUCHE S. - Contribution à l'étude du transport solide par l'Oued Zéroud à Sidi-Saâd, Mémoire de Fin d'Etudes du Cycle de Spécialisation, I.N.A.T., 1982.

[3] BORLAND W. M. - * Reservoir Sedimentation ", in River Mechanics, H. W. Shen, ed., Water Resources Publications, Fort Collins, Colorado, 1971.

[4] Chauvel A. - Etude Pédologique du Périmètre de l'oued Chiba, N*187, Tunis, Service Pédologique, 1961.

[5] CHow V. T. - Handbook of Applied Hydrology: A Compedium of Water Resources Technology, McGrawHill, 1964.

[6] CHow V. T. - Open Channel Hydraulics, McGraw-Hill, 1959.

[7] Claude J., Chartier R. - Mesure de l'envasement dans les retenues de six barrages en Tunisie, Campagne de 1975, Office de la Recherche Scientifique et Technique OutreMer.

[8] Kchouk A., Masmoudi A. - Protection de Nabeul contre les inondations, Projet de Diplôme, Ecole Nationale d'Ingénieurs de Tunis, 1983.

[9] LARRAS J. - Hydraulique et granulats, Eyrolles, 1972.

[10] Linsley R. K., Kohlev M. A., Paulhus J. L. H. - Hydrology for Engineers, McGraw-Hill, 1975.

[11] Meyer L. D. - «Soil Erosion by Water on Upland Areas ", in River Mechanics, H. W. Shen, ed., Water Resources Publications, Colorado.

[12] Simons D. B., SưnturK F. - Sediment Transport Technology, Water Resources Publications, Fort Collins, Colorado, 1977.

[13] WischmeIer W. H., SMITH D. D. — $\approx$ Soil-loss Estimation as a Tool in Soil and Water Management Planning ", AIHS, Colloque de Bari, du 1-10 au 8-10, 1962. 